\title{
Initiation à la conception d'antennes Yagi-Uda pour des lycéens
}

\author{
S. Avrillon \\ IETR et pôle CNFM de Rennes (CCMO), Université de Rennes 1, Rennes, France \\ Contact email : stephane.avrillon@univ-rennes1.fr r
}

\begin{abstract}
Dans le cadre des actions de sensibilisation vers l'enseignement secondaire du projet ANR FINMINA [2], une initiation à la conception d'antennes est proposée aux lycées de l'Académie de Rennes. Dans ce cadre, depuis 2016, plusieurs groupes d'élèves sont venus dans les salles de travaux pratiques de la plateforme électronique de l'Université de Rennes 1 pendant deux demijournées afin de dimensionner, simuler, réaliser et mesurer une antenne Yagi pour des applications WiFi.
\end{abstract}

\section{Le contexte de cette initiation}

Les lycéens sont de grands utilisateurs de systèmes de télécommunication, que ce soit pour la téléphonie mobile ou l'internet. Ils connaissent en général parfaitement les applications liées à ces nouvelles technologies mais sont peu au fait des technologies qui se cachent derrière ces objets communicants, en particulier l'électronique liée aux télécommunications. L'objectif de ces actions envers les lycéens est de leur montrer que ces objets fonctionnent grâce à des composants électroniques et qu'il existe des formations à l'Université qui permettent d'aller vers des métiers liés à la conception des systèmes électronique, en particulier de télécommunication.

En deux demi-journées, l'idée est de concevoir, réaliser et tester une antenne de type Yagi-Uda pour le WiFi. Le choix d'étudier ce composant a été fait car c'est un élément à la fois complexe d'un point de vue scientifique, faisant intervenir des notions d'ondes et d'électromagnétisme liées au programme de physique de lycée, et assez facile à réaliser d'un point de vue technologique. Ces deux demi-journées permettent aussi aux élèves de comprendre la démarche scientifique allant de la recherche bibliographique, à la simulation puis la réalisation et enfin la confrontation entre la mesure et les performances initialement prévues.

\section{La démarche de conception}

Il est intéressant de faire comprendre aux lycéens que la démarche qui est employée sur les deux demi-journées est une démarche classique pour la conception de n'importe quel objet technique :

- Enoncé des spécifications souhaitées

- Recherche bibliographique et pré-dimensionnement,

- Conception assistée par ordinateur,

- Réalisation d'un prototype,

- Mesure du prototype,

- Eventuelles améliorations à l'aide de rétro-simulation.

Spécifications souhaitées

La première étape consiste à définir des spécifications pour l'antenne que l'on souhaite réaliser. Ici, les spécifications sont volontairement peu restrictives. Il est expliqué aux 
lycéens que l'antenne devra être utilisée pour la bande WiFi (autour de 2,45GHz) dans des cas où la borne WiFi est éloignée. Après discussion, on arrive à la conclusion qu'une antenne directive (qui envoie et reçoit l'énergie dans une direction privilégiée) est nécessaire.

\section{$\underline{\text { Recherche bibliographique }}$}

Cette étape, dans le cadre des deux demi-journées, est volontairement réduite car elle peut être très chronophage. La conception de l'antenne repose sur une étude d'un document issu d'un ouvrage de référence sur les antennes, "Antenna Theory » de C. Balanis [1]. L'antenne choisie est la «Yagi-Uda», autrement appelée communément «antenne râteau ».

Constituée de fils conducteurs, sa réalisation est assez simple. Comme le montre la Fig.1, elle est constituée d'un élément actif (Driven element), d'un brin réflecteur et de plusieurs brins directeurs. L'élément actif utilisé ici est un dipôle replié. . Il est alimenté par un connecteur SMA sur lequel on pourra envoyer un signal WiFi ou un signal issu d'un générateur de signaux haute fréquence permettant de fournir des fréquences autour de 2,45GHz. On choisit une antenne Yagi à 5 éléments, donc avec 3 brins directeurs.

La Fig. 2 donne les dimensions du brin réflecteur $\left(l_{1}\right)$ et des brins directeurs $\left(l_{3}\right.$ à $\left.l_{5}\right)$ de l'antenne, le diamètre des brins $(d)$ et les espaces entre les brins $\left(s_{i j}\right)$. Toutes ces valeurs sont données en fonction de la longueur d'onde.

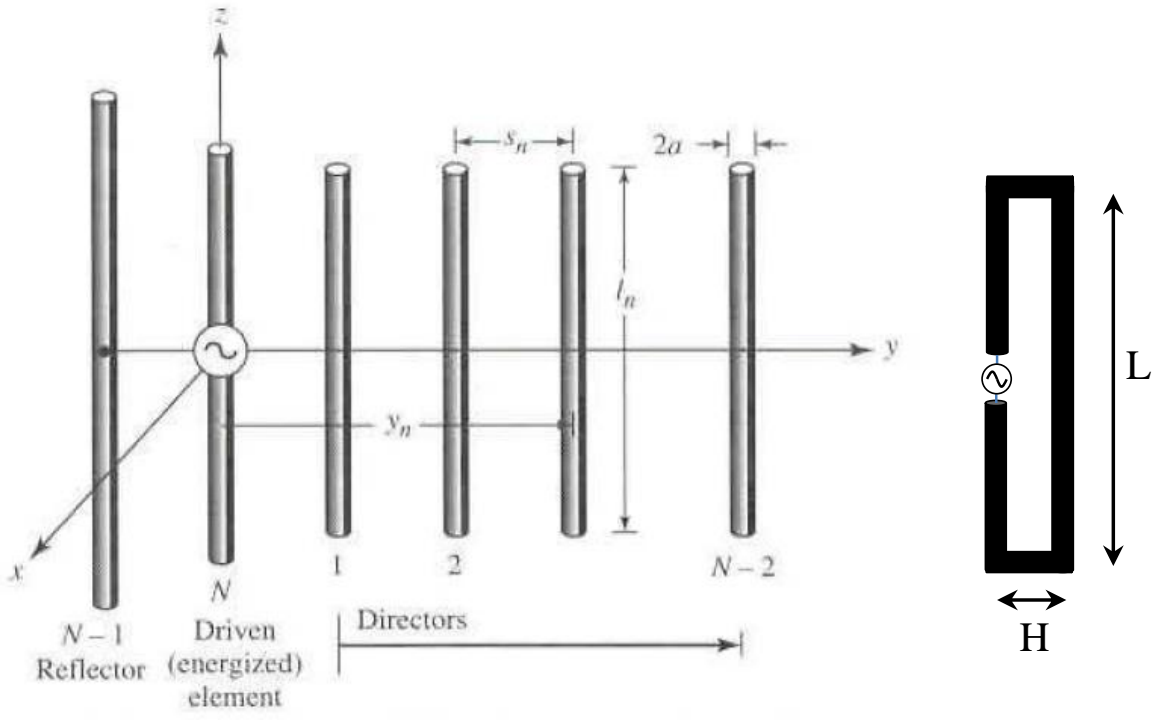

Fig.1. À droite - Constitution d'une antenne Yagi-Uda, extrait de Antenna Theory de C. Balanis [1], à gauche - Dimension du dipole replié servant d'élément actif à l'antenne

\section{Détermination des dimensions de l'antenne pour le WiFi}

La Fig.1 étant associée au tableau (Fig.2) donnant les dimensions en fonction de la longueur d'onde $\lambda$, les élèves doivent donc, dans un premier temps, calculer $\lambda$ en utilisant la formule (1).

$$
\lambda=\frac{c}{f}=\frac{310^{8}}{2.4510^{9}}=12,2 \mathrm{~cm}
$$

Ce calcul de $\lambda$ permet de dimensionner l'ensemble de l'antenne Yagi, avec :

- le diamètre des fils $\mathrm{d}=0,008512,2 \mathrm{~cm}=1 \mathrm{~mm}$,

- la longueur du brin réflecteur $l_{1}=0,48212,2 \mathrm{~cm}=5,9 \mathrm{~cm}$

- la longueur du premier brin directeur $l_{3}=0,48212,2 \mathrm{~cm}=5,24 \mathrm{~cm}$ 
- la longueur du deuxième brin directeur $l_{4}=0,42412,2 \mathrm{~cm}=5,19 \mathrm{~cm}$

- la longueur du troisième brin directeur $1_{5}=0,48212,2 \mathrm{~cm}=5,24 \mathrm{~cm}$

- $\quad$ 'espacement entre les brins $\mathrm{s}_{12}=\mathrm{s}_{\mathrm{ik}}=0,212,2 \mathrm{~cm}=2.45 \mathrm{~cm}$

Les dimensions du dipôle replié doivent être ajustées afin que le courant sur ce dipôle ait un mode de résonance à la fréquence $2,45 \mathrm{GHz}$. Dans un premier temps, on prend une forme rectangulaire avec un côté de $\mathrm{L}=5,6 \mathrm{~cm}$ et une hauteur de $\mathrm{H}=0,5 \mathrm{~cm}$, ce qui fait une longueur totale de $12,2 \mathrm{~cm}$, soit une longueur d'onde.

La Fig.2 donne aussi une idée de la directivité de l'antenne (directivity relative to halfwave dipole). Pour une antenne à 5 brins, la directivité est de $9,2 \mathrm{~dB}_{\mathrm{d}}$. Cela veut dire que cette antenne rayonne $9,2 \mathrm{~dB}$ de plus qu'une antenne dipôle $\lambda / 2$ dans la direction où le rayonnement est maximal. Cette unité, le $\mathrm{dB}_{\mathrm{d}}$ (par rapport à un dipôle $\lambda / 2$ ), n'est pas facile à comprendre et il est plus simple de le convertir en $\mathrm{dB}_{\mathrm{i}}$ (par rapport à une antenne isotrope) avec $x d_{B}=x d B_{d}+2,15 d B$. Ainsi, notre antenne a un gain de $11,35 \mathrm{~dB}_{\mathrm{i}}$. Cela veut dire que si on alimente notre antenne avec une puissance donnée, elle rayonnera dans sa direction privilégiée $11,35 \mathrm{~dB}$ de plus (soit 13,64 fois plus de puissance) que si on alimentait une antenne omnidirectionnelle (isotrope) avec la même puissance.

\begin{tabular}{|c|c|c|c|c|c|c|c|}
\hline \multirow{2}{*}{\multicolumn{2}{|c|}{$\begin{array}{l}d / \lambda=0.0085 \\
s_{12}=0.2 \lambda\end{array}$}} & \multicolumn{6}{|c|}{ LENGTH OF YAGI-UDA (IN WAVELENGTHS) } \\
\hline & & 0.4 & 0.8 & 1.20 & 2.2 & 3.2 & 4.2 \\
\hline \multicolumn{2}{|c|}{$\begin{array}{l}\text { LENGTH OF } \\
\text { REFLECTOR }\left(l_{1} / \lambda\right)\end{array}$} & 0.482 & 0.482 & 0.482 & 0.482 & 0.482 & 0.475 \\
\hline \multirow{15}{*}{ 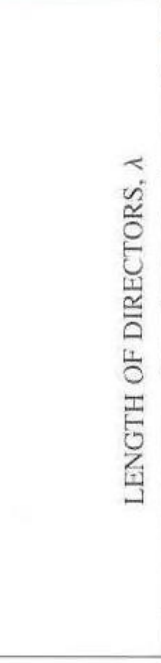 } & $l_{3}$ & 0.442 & 0.428 & 0.428 & 0.432 & 0.428 & 0.424 \\
\hline & $l_{4}$ & & 0.424 & 0.420 & 0.415 & 0.420 & 0.424 \\
\hline & $l_{5}$ & & 0.428 & 0.420 & 0.407 & 0.407 & 0.420 \\
\hline & $l_{6}$ & & & 0.428 & 0.398 & 0.398 & 0.407 \\
\hline & $I_{7}$ & & & & 0.390 & 0.394 & 0.403 \\
\hline & $l_{8}$ & & & & 0.390 & 0.390 & 0.398 \\
\hline & $l_{9}$ & & & & 0.390 & 0.386 & 0.394 \\
\hline & $l_{10}$ & & & & 0.390 & 0.386 & 0.390 \\
\hline & $l_{11}$ & & & & 0.398 & 0.386 & 0.390 \\
\hline & $l_{12}$ & & & & 0.407 & 0.386 & 0.390 \\
\hline & $l_{13}$ & & & & & 0.386 & 0.390 \\
\hline & $l_{14}$ & & & & & 0.386 & 0.390 \\
\hline & $I_{15}$ & & & & & 0.386 & 0.390 \\
\hline & $I_{16}$ & & & & & 0.386 & \\
\hline & $l_{17}$ & & & & & 0.386 & \\
\hline \multicolumn{2}{|c|}{$\begin{array}{l}\text { SPACING BETWEEN } \\
\text { DIRECTORS }\left(s_{i k} / \lambda\right)\end{array}$} & 0.20 & 0.20 & 0.25 & 0.20 & 0.20 & 0.308 \\
\hline \multicolumn{2}{|c|}{$\begin{array}{l}\text { DIRECTIVITY RELATIVE } \\
\text { TO HALF-WAVE } \\
\text { DIPOLE (dB) }\end{array}$} & 7.1 & 9.2 & 10.2 & 12.25 & 13.4 & 14.2 \\
\hline \multicolumn{2}{|c|}{$\begin{array}{l}\text { DESIGN CURVE } \\
\text { (SEE FIGURE } 10.25)\end{array}$} & (A) & (B) & (B) & (C) & (B) & (D) \\
\hline
\end{tabular}

Fig.2. Tableau donnant les dimensions de l'antenne Yagi en fonction de la longueur d'onde $\lambda$

\section{Simulation de l'antenne sous 4NEC2}

Une fois les dimensions déterminées, la deuxième étape est de vérifier par simulation que les performances de l'antenne sont bien conformes à ce que l'on attend, à savoir :

- l'antenne fonctionne autour de $2,45 \mathrm{GHz}$ pour le $\mathrm{WiFi}$ 
- le rayonnement de l'antenne est directif avec un gain proche de celui attendu : $11,35 \mathrm{dBi}$.

Pour cela, on utilise un logiciel de simulation électromagnétique, 4NEC2 [3], qui est gratuit. Ce logiciel est assez simple d'utilisation. Il faut dessiner sur une interface graphique les fils qui constituent l'antenne, définir la fréquence $2,45 \mathrm{GHz}$ et placer sur le dipôle replié qui sert d'élément actif une source de tension comme le montre la Fig.3.

L'icône «calculatrice" permet d'ouvrir une fenêtre dédiée aux paramètres de simulation. Il est possible de faire trois types de simulation. «Far Field pattern » permet de calculer le champ lointain électromagnétique rayonné par l'antenne dans toutes les directions à une fréquence donnée. «Frequency sweep» permet de vérifier le comportement de l'antenne sur une plage de fréquence. «Near Field pattern » permet de calculer le champ proche. Cette dernière simulation ne sera pas utilisée dans notre cas.

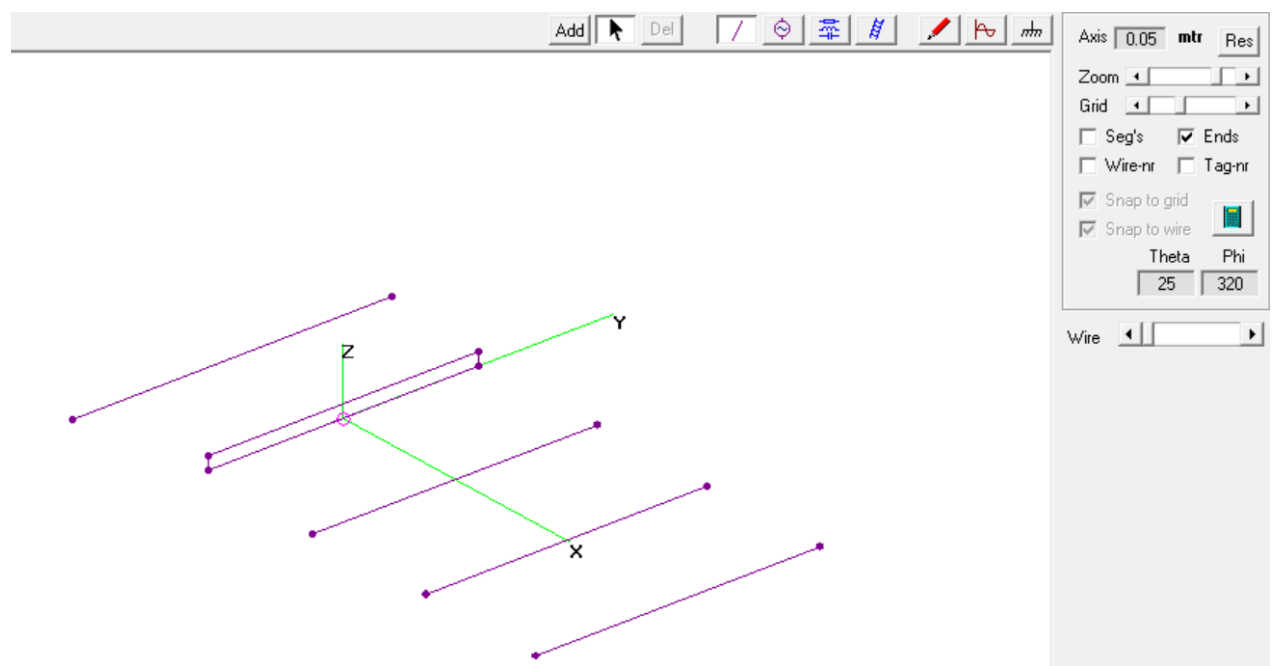

Fig.3. Interface graphique du logiciel 4NEC2 avec l'antenne Yagi à 5 éléments. La source correspond au cercle rouge placé au centre du repère xyz

La première simulation est réalisée dans le mode «Frequency Sweep » afin de voir si l'antenne est bien dimensionnée pour une application WiFi. Pour cela, on règle la plage de fréquence de simulation de $2.000 \mathrm{MHz}$ à $3.000 \mathrm{MHz}$ avec un pas de $5 \mathrm{MHz}$. La Fig.4 à gauche donne le coefficient de réflexion en fonction de la fréquence. En dessous de $-10 \mathrm{~dB}$, au moins $90 \%$ de la puissance envoyée dans l'antenne est rayonnée et moins de $10 \%$ est renvoyée (réfléchie) vers le générateur. On remarque que l'antenne telle que dimensionnée ne fonctionne pas correctement. Le niveau du coefficient de réflexion est trop haut (supérieur à $-8 \mathrm{~dB}$ ). De plus, on remarque que le coefficient de réflexion est meilleur autour de $2.300 \mathrm{MHz}$ et donc trop bas en fréquence. Le dipôle fonctionne donc trop bas en fréquence et ses dimensions doivent être ajustées. On voit aussi une fréquence où il semble y avoir une résonance à $2.565 \mathrm{MHz}$. Cette fréquence est sans doute due à la résonance des brins directeurs.

Le travail des lycéens consiste alors à modifier de façon appropriée le dipôle replié afin que sa longueur totale diminue et que sa fréquence de résonance augmente. Pour faire cela, on peut jouer sur la hauteur du dipôle et/ou sur sa longueur. En simplifiant, la fréquence est inversement proportionnelle à la longueur totale du dipôle. Comme la fréquence est 1,065 fois trop petite $(1,065=2.450 \mathrm{MHz} / 2.300 \mathrm{MHz})$, il faut réduire le dipôle de $12,2 \mathrm{~cm}$ à $11,45 \mathrm{~cm}$. En faisant cela, on se rend compte que la fréquence est toujours trop basse à 2,41GHz. Il faut donc encore réduire la longueur du dipôle à $\mathrm{L}=5,0 \mathrm{~cm}$, on a alors une 
longueur totale du dipôle de $11 \mathrm{~cm}$ et on obtient le coefficient de réflexion de la Fig.4 à droite. On voit alors que la fréquence optimale est bien $2.45 \mathrm{GHz}$ avec un coefficient de réflexion d'environ $-20 \mathrm{~dB}$ (soit $1 \%$ de puissance réfléchie).
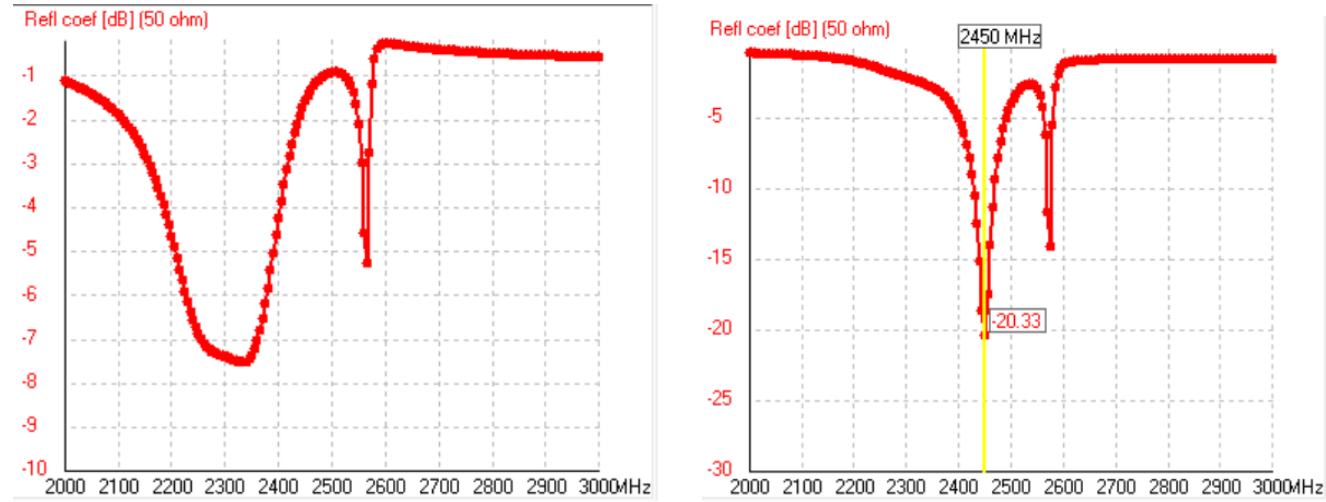

Fig.4. Coefficient de réflexion simulé par 4NEC2 de l'antenne Yagi. A gauche, $\mathrm{L}=5,6 \mathrm{~cm}$ et $\mathrm{H}=0,5 \mathrm{~cm}$. A droite, $\mathrm{L}=5 \mathrm{~cm}$ et $\mathrm{H}=0,5 \mathrm{~cm}$

Suivant le temps disponible, on peut demander aux lycéens de tester d'autres dimensions de dipôle avec la même fréquence optimale $2,45 \mathrm{GHz}$. On peut alors trouver : $\mathrm{L}=4,7 \mathrm{~cm}$ et $\mathrm{H}=1 \mathrm{~cm}, \mathrm{~L}=4,4 \mathrm{~cm}, \mathrm{H}=1,5 \mathrm{~cm}$. Comme le montre la Fig.5, on remarque que cela modifie très peu le comportement fréquentiel de l'antenne.
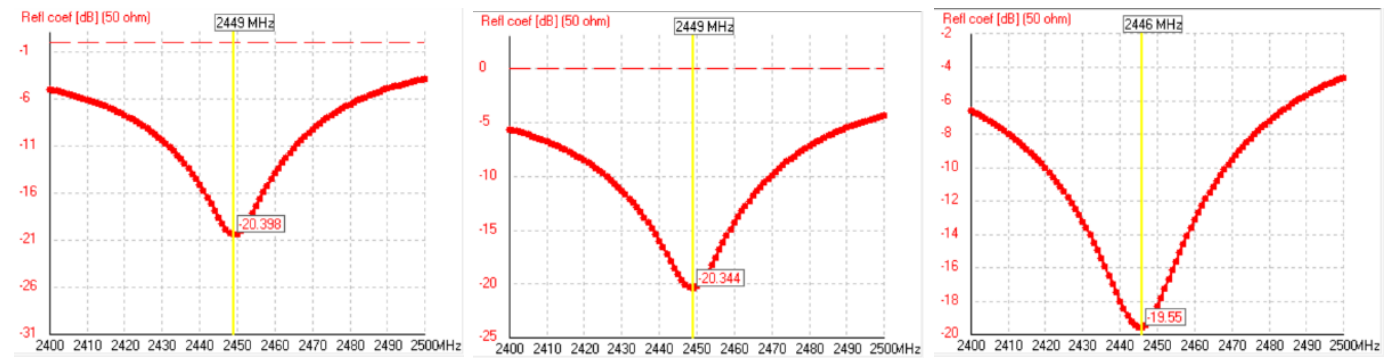

Fig.5. Coefficient de réflexion simulé par 4NEC2 de l'antenne Yagi pour des dipôles repliés de dimensions différentes : de gauche à droite : $\mathrm{L}=5 \mathrm{~cm}$ et $\mathrm{H}=0,5 \mathrm{~cm}, \mathrm{~L}=4,7 \mathrm{~cm}$ et $\mathrm{H}=1 \mathrm{~cm}, \mathrm{~L}=4,4 \mathrm{~cm}$ et $\mathrm{H}=1,5 \mathrm{~cm}$.

Le deuxième type de simulation est réalisée dans le mode «Far Field pattern ». On reprend les dimensions $\mathrm{L}=5 \mathrm{~cm}$ et $\mathrm{H}=0,5 \mathrm{~cm}$ pour visualiser en mode $3 \mathrm{D}$ l'antenne et son diagramme de rayonnement, comme le montre la Fig.6. On remarque que l'énergie rayonnée est bien dirigée vers l'avant de l'antenne correspondant aux brins directeurs. On remarque qu'il y a aussi de la puissance rayonnée à la verticale. Cela est dû à l'orientation du dipôle replié. Il est éventuellement possible de le placer horizontalement afin d'améliorer le diagramme de rayonnement mais cela complique un peu la réalisation de l'antenne par la suite. Le gain simulé de l'antenne est de $11,1 \mathrm{dBi}$, donc proche des $11,35 \mathrm{dBi}$ théoriques.

On peut aussi visualiser le diagramme de rayonnement suivant différentes coupes, en particulier les coupes horizontales (plan $\mathrm{xOz}$ ) et verticale (plan xOy) comme le montre la Fig.7. On remarque sur le plan vertical que l'antenne possède une ouverture plus grande que dans le plan horizontal. 


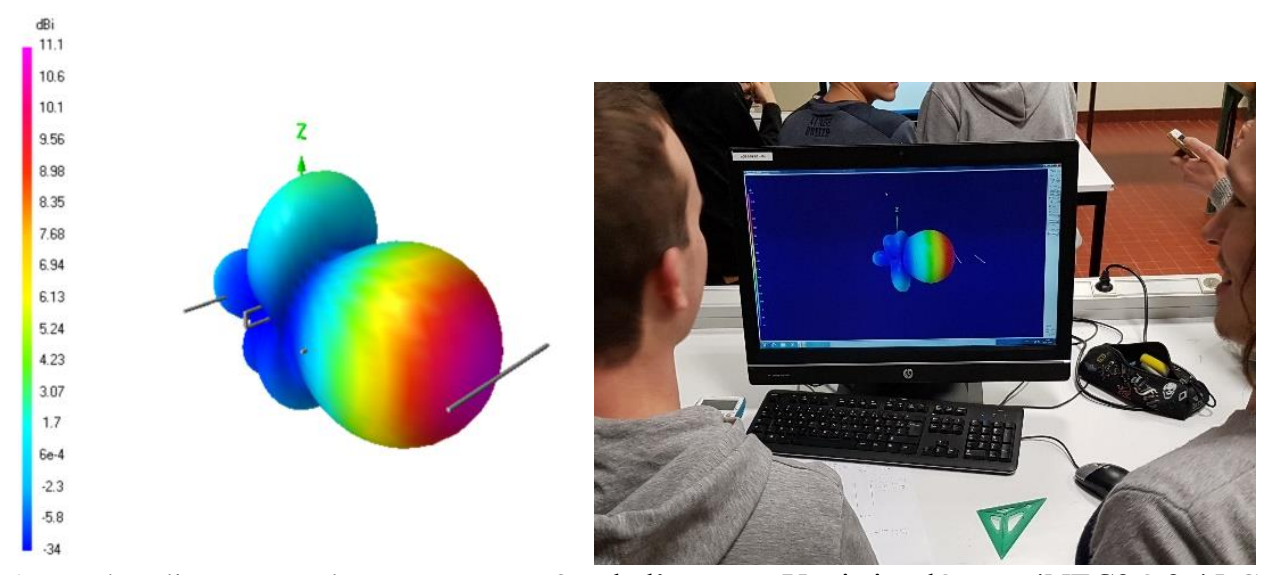

Fig.6. A gauche, diagramme de rayonnement 3D de l'antenne Yagi simulée par 4NEC2 à $2.45 \mathrm{GHz}$. A droite, vérification du rayonnement par simulation sur 4NEC2 par un binôme de lycéens.
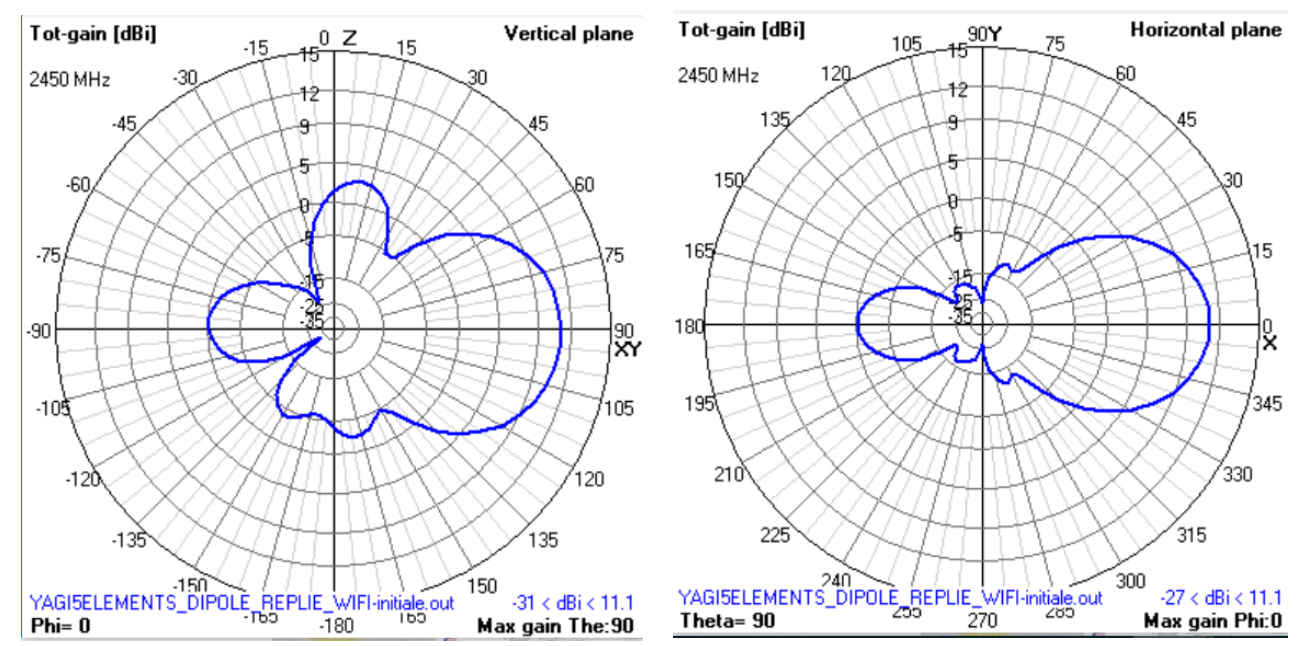

Fig.7. Diagramme de rayonnement de l'antenne Yagi simulée par 4NEC2 à 2,45GHz dans le plan vertical à gauche et horizontal à droite.

\section{Réalisation de l'antenne Yagi}

Pour réaliser l'antenne, les élèves ont à disposition du fil de cuivre émaillé de diamètre $1,05 \mathrm{~mm}$, un morceau de Rohacellß de section $10 \mathrm{~mm} \times 10 \mathrm{~mm}$, une perceuse avec un foret $1 \mathrm{~mm}$, une pince coupante, un fer à souder, une machine de désémaillage (Abisofix) et un câble coaxial $50 \Omega$ de $50 \mathrm{~cm}$ avec un connecteur SMA comme le montre la Fig. 8 à gauche. Le Rohacell ${ }^{\circledR}$ est une mousse qui a un comportement électromagnétique (permittivité et perméabilité) très proche de celui de l'air, ce qui permet d'avoir un support pour les brins qui est «transparent» d'un point de vue électromagnétique.

Les lycéens découpent dans un premier temps les différents brins de l'antenne dans le fil de cuivre en respectant les dimensions calculées précédemment. Dans un second temps, ils découpent le Rohacell ${ }^{\circledR}$ qui servira de support aux brins et y percent des trous afin de passer les brins à des intervalles respectant les espacements entre brins. La partie la plus délicate est la réalisation du dipôle replié. Le brin qui sert au dipôle replié est dans un premier temps désémaillé à ses extrémités afin de faciliter par la suite l'étamage. Il est ensuite inséré dans le support Rohacell ${ }^{\circledR}$ avant le pliage réalisé avec des pinces. Après pliage du dipôle, les extrémités sont étamées avec un fer à souder ayant une grosse panne puis le câble SMA est soudé à celui-ci. La Fig.9 montre des lycéens à différentes étapes de la fabrication de l'antenne Yagi. La Fig. 8 à droite montre une photo d'antenne Yagi réalisée par les lycéens. 

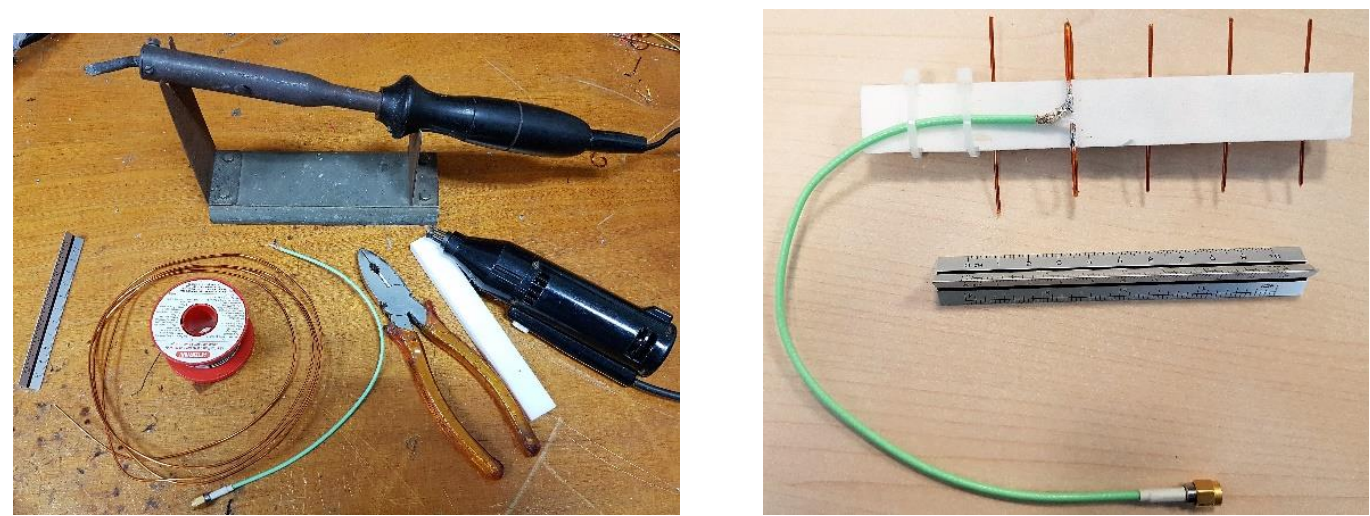

Fig.8. A gauche, matériel nécessaire pour la réalisation de l'antenne. A droite, photo d'une antenne Yagi réalisée par les lycéens.

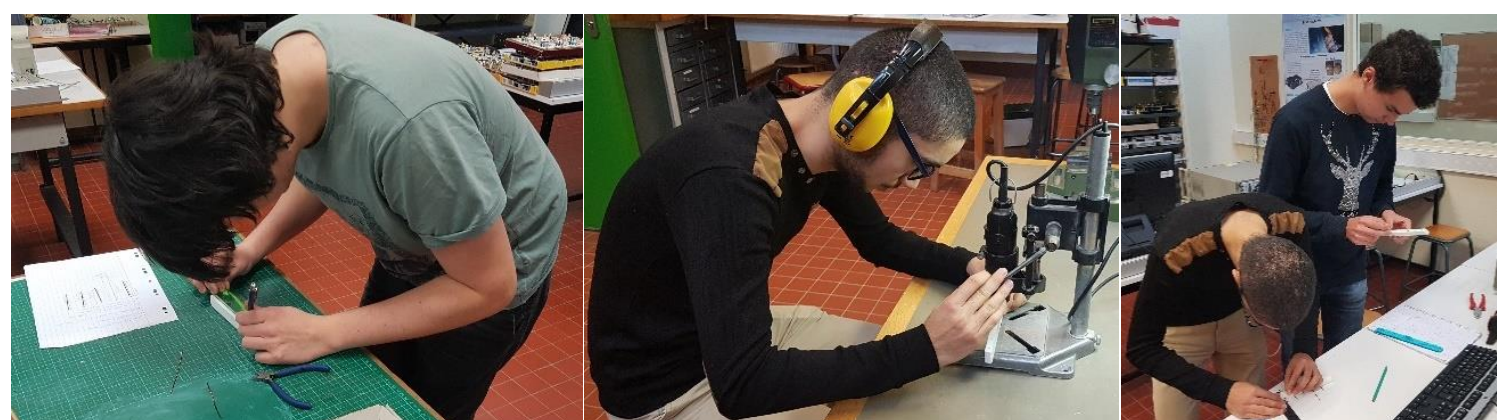

Fig.9. Quelques lycéens en cours de fabrication de l'antenne Yagi : découpage des brins, perçage du support et assemblage.

\section{Mesure de l'antenne Yagi}

Pour mesurer l'antenne réalisée, nous avons en salle de TP des équipements spécifiques :

- $\quad$ un analyseur de réseau qui permet de mesurer le coefficient de réflexion,

- une chambre anéchoïde.

Le coefficient de réflexion est mesuré (Fig.10) sur table avec un analyseur de réseau. Les conditions de mesure de ne sont pas optimales car les ondes sont perturbées par les objets situés à proximité (ordinateur, appareil de mesure, table...). Malgré tout, cela permet d'avoir une bonne idée de la fréquence pour laquelle l'antenne rayonne. On peut le vérifier en passant la main devant l'antenne et en regardant le coefficient de réflexion modifié en temps réel. Sur le résultat de mesure, on remarque ici que le coefficient de réflexion n'est pas identique à celui simulé sur 4NEC2 (Fig.4). Ceci est dû à la qualité de la réalisation, en particulier les longueurs de brin qui ne sont pas parfaite, le diamètre des brins qui est aussi différent, au pliage du dipôle qui n'est pas identique à celui de la simulation et aux perturbations des objets aux alentours. Néanmoins, on peut noter qu'à la fréquence $2,45 \mathrm{GHz}$, le coefficient de réflexion est quasiment de $-12 \mathrm{~dB}$, ce qui veut dire que $6,3 \%$ de la puissance envoyée sur le port de l'antenne est réfléchie et $93,7 \%$ est rayonnée. Ceci est tout à fait correct. 

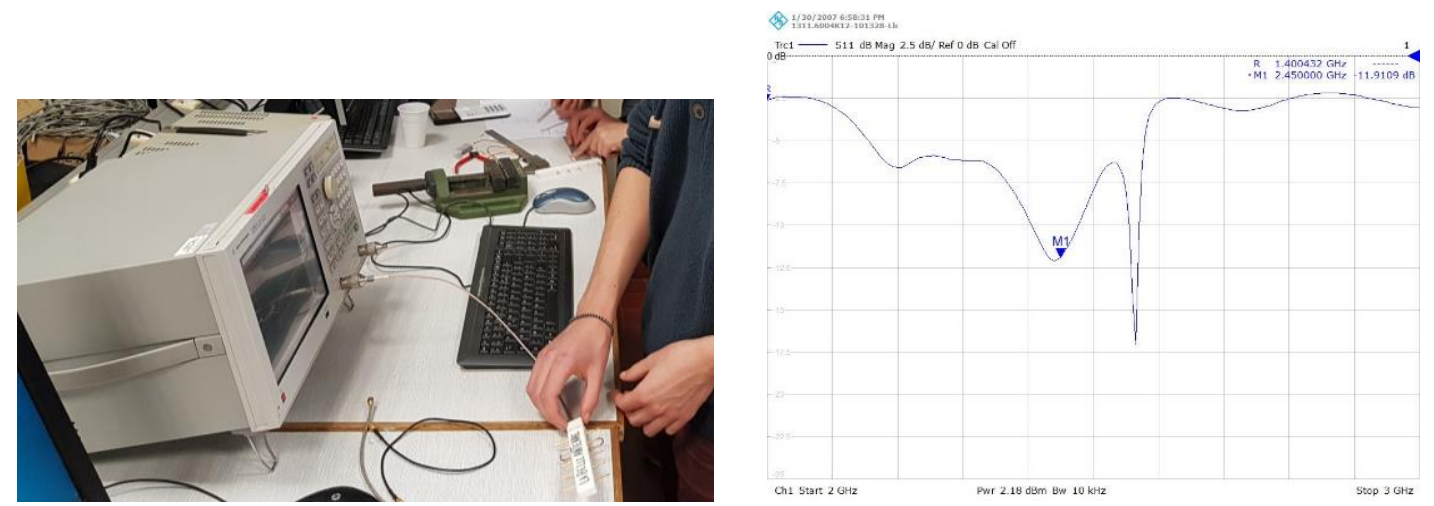

Fig.10. A gauche, la mesure du coefficient de réflexion sur table avec un analyseur de réseau. A droite, une copie d'écran de la mesure réalisée pour l'antenne Yagi réalisée.

Pour les mesures de rayonnement, les murs de la chambre anéchoïde sont entièrement métallisés afin que la mesure soit isolée électromagnétiquement de l'extérieur et sont recouverts d'absorbants électromagnétiques afin d'éviter les multiples rebonds des ondes à l'intérieur de la chambre. Une antenne de réception est placée au fond de la pièce. C'est une antenne de référence dont on connait les caractéristiques de rayonnement. A l'avant de la pièce, proche de la porte, à l'émission, l'antenne sous test est fixée sur un support tournant. Un analyseur de réseau permet de mesurer le rapport entre la puissance émise et la puissance reçu en fonction de l'angle de rotation de l'antenne sous test. L'analyseur de réseau et le support tournant sont pilotés via un logiciel spécialisé qui en déduit le diagramme de rayonnement de l'antenne sous test.

Le diagramme de rayonnement est ensuite mesuré à la fréquence optimale. La Fig.11 à gauche montre un lycéen dans la chambre anéchoïde en train de fixer son antenne Yagi sur le support tournant. A droite, les diagrammes de rayonnement mesurés et simulés avec 4NEC2 sont donnés sur le même graphique. On remarque que le lobe principal du diagramme de rayonnement, situé en face de l'antenne à un angle de $0^{\circ}$, possède à peu près les mêmes caractéristiques : le gain mesuré est légèrement inférieur, 10,7 dBi contre 11,35 dBi simulé et la largeur du lobe quasiment identique. Par contre, en dehors du lobe principal, on remarque des différences et en particulier que le diagramme mesuré n'est pas symétrique contrairement à celui simulé. Cela montre que la réalisation de l'antenne n'est pas parfaite: on peut d'ailleurs noter que le dipôle replié n'est pas alimenté de façon symétrique et qu'il faudrait ajouter un symétriseur pour améliorer le diagramme mesuré.

Néanmoins, la fonction de l'antenne est bien respectée avec un rayonnement principalement dirigée vers l'avant de l'antenne permettant d'avoir un gain d'antenne supérieur à $10 \mathrm{dBi}$.
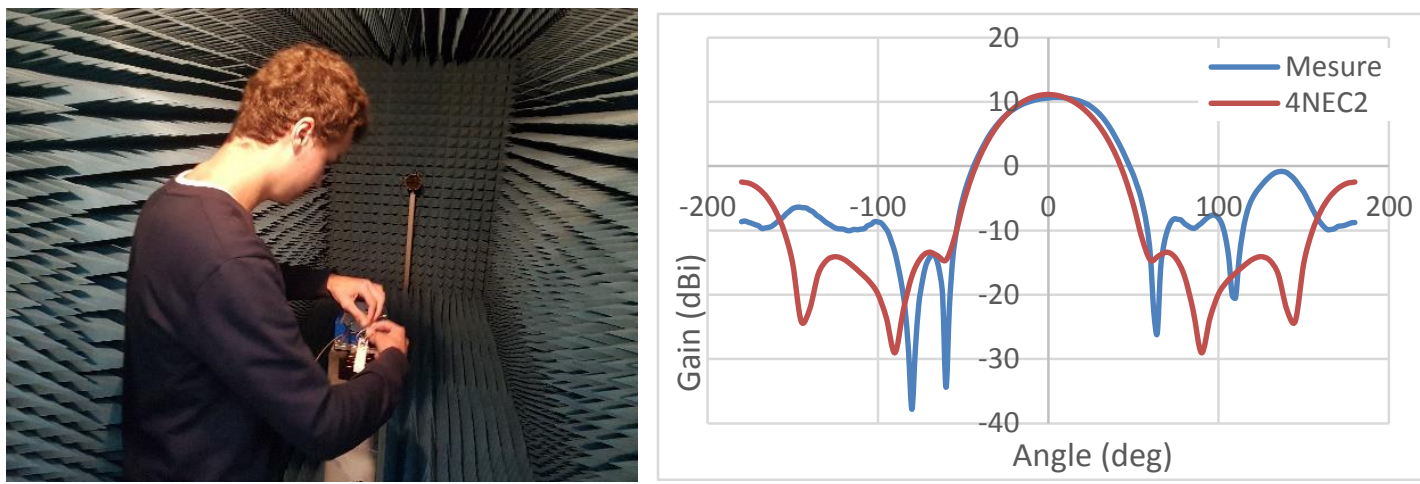

Fig.11. A gauche, lycéen dans la chambre anéchoïde en train de fixer son antenne Yagi avant la mesure et, à droite, la mesure réalisée comparée à la simulation sur 4NEC2. 


\section{Remerciements}

L'accueil de ces lycéens est fait dans le cadre du projet FINMINA et l'auteur tient donc à remercier le soutien de ce programme [2]. Il tient aussi à remercier les élèves et les enseignants du lycée Beaumont de Redon qui ont participé tous les ans à ces TP et qui ont réalisé les antennes présentées dans cet article.

\section{Références}

1. C. Balanis in Antenna Theory : Analysis and Design, p. 514, Wiley Editor, $2^{\text {nd }}$ Edition, 2005.

2. IDEFI-FINMINA : Initiative d'Excellence - Formation Innovante en MIcroélectronique et Nanotechnologies, ANR-11-IDFI-0017. Website: http://www.cnfm.fr/VersionFrancaise/actualites/ FINMINA.htm (Accès juillet 2018)

3. 4NEC2, https://www.qsl.net/4nec2/ 